\title{
Efficient Cruise Control of Electric Motorcycle Using PID
}

\author{
Rohan Sawant and Andrew Kidd \\ R. Sawant \\ Vidyalankar Institute of Technology, Mumbai, India \\ e-mail: rohansawant96@gmail.com
}

\author{
A. Kidd \\ Teesside University, Middlesbrough, UK \\ e-mail: A.Kidd@tees.ac.uk
}

\begin{abstract}
The automotive industry is progressing expeditiously. The popularity of electric vehicles (EV) has shot up over the last few years. As a matter of fact, electric drive systems such as DC motor drives, one of the electrical drives, are rapidly gaining prominence because of their high efficiency, better dynamic response and low upkeep. The following work deals with the drivetrain of the pre-existing electric motorcycle comprised of a brushed DC motor. In this work, a system is developed to maintain the speed of the electric motorcycle at a set speed, under various disturbances using PI control. A detailed mathematical model, transfer function and simulation of the same are obtained using the software package MATLAB, SIMULINK. The controlled DC motor is made to track a variable speed set point with zero steady-state error and desirable disturbance rejection capabilities.
\end{abstract}

Keywords DC motor $\cdot$ PID $\cdot$ Matlab

\section{Introduction}

DC motor drives play a significant role in mechanical and other applications such as steel moving plants, electric trains and robotics. For the most part, an elite engine drive framework must have great powerful speed order and load controlling reaction to perform undertaking. DC drives, due to their straightforwardness, simplicity of utilization, dependability and favourable cost have for quite some time been a spine of mechanical and robotic applications where speed and position control of motor are required. DC drives are less complex, as when it comes to motorcycles, the power conversion factor from DC to AC is eliminated. Also, the speed-torque attributes of a DC motor are robust [1]. 
Although three-phase induction motors are the most widely recognized sort of motors utilized in industrial applications, DC motors are expanding in prevalence because of their execution points of interest over AC motors for applications ranging from high-speed automation to electric motorbikes. Applications where speed should be varied, or torque should be controlled with high exactness, brushed DC motors are used. Today, locomotives are being offered with powered gadgets, in addition designers are seeking to improve the performance of the equipment they design, which often entails upgrading from AC motor to a DC motor, specifically, places where increasing accelerations are required. DC drives are additionally being fabricated in regularly expanding volumes for much Bijou applications, such as solar-based gadgets, toys and cell phone handsets [2].

In this work, a corresponding DC motor similar to the one used in an electric motorcycle is considered. A transfer function model of the motor is obtained using the reaction curve method and analysed in MATLAB, compared to physical data from the test motor to check for goodness-of-fit. Further using this model and the direct design synthesis (DDS) control design approach, the speed of the motor will be made to track a variable set point and then be maintained under the influence of disturbances, such as a change in the inclination of the surface the bike is travelling on (i.e. up/down a hill) (Fig. 1).

\section{Mathematical Modelling}

\subsection{Transfer Function Derivation of a DC Motor (Bottom-Up Approach)}

A separately excited DC motor system is considered. To obtain the mathematical model of the same, consider, supply voltage ' $v$ ' applied to the motor circuit (refer Fig. 2), and the motor generates torque $(T)$ that is proportional to the product of the

Fig. 1 DC motor equivalent circuit

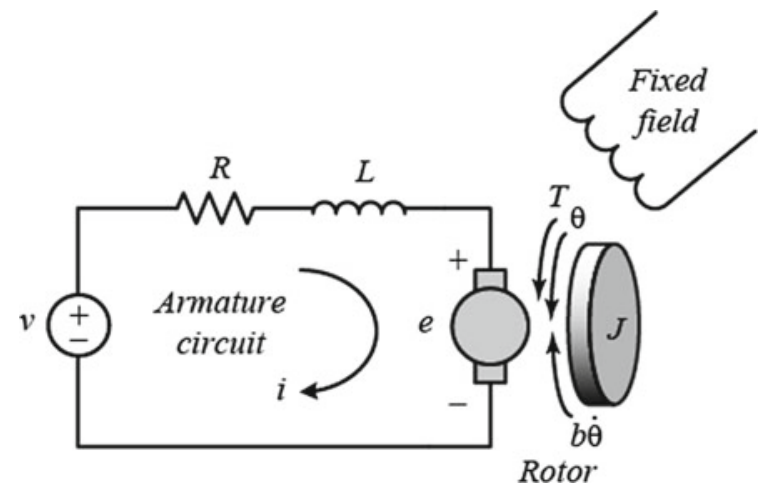


magnetic field flux ' $\phi$ ' and the rotor armature current ' $i$ ',

$$
T=K_{T} i \phi
$$

The Back EMF is a counter voltage that is proportional to the product of magnetic field flux $(\phi)$ and the rotor angular velocity $(\omega)$,

$$
e=K_{E} \phi \omega
$$

Making use of KVL voltage law in the above motor circuit,

$$
v=R i+L \frac{\mathrm{d} i}{\mathrm{~d} t}+e
$$

Considering the above fundamental equations into Laplace transform, respectively, and further simplifying it [3,4], we get the first-order transfer function model of the DC motor as,

$$
\frac{\Omega(s)}{v(s)}=\frac{\frac{1}{K_{E}}}{1+\tau_{m} s}
$$

where $\tau_{m}=$ Electromechanical time constant.

\subsection{System Identification-Reaction Curve Method}

The first-order plus time-delay (FOPTD) model has been widely used to design and implement the process controllers of the system. The terms related to the low-order plus time-delay processes are very useful for describing the dynamic characteristics of given process. The FOPTD process derived for DC motor system is given in Eq. (1).

From Eq. (1), it can be realized that $K$ and $\tau$ are called the static gain and the time constant, respectively. The step input applied to the motor is $\Delta u$, and the maximum rated voltage under which the motor operates is represented by $\Delta y$. Using the formulae, the terms $K, \tau$ and $d$ are computed.

$$
\begin{gathered}
K=\frac{\Delta y}{\Delta u} \quad(\text { System Gain) } \\
\tau=1.5\left(t_{2}-t_{1}\right) \quad \text { (Time Constant) } \\
d=1.5\left(t_{1}-\frac{t_{2}}{3}\right) \quad \text { (Time delay) }
\end{gathered}
$$




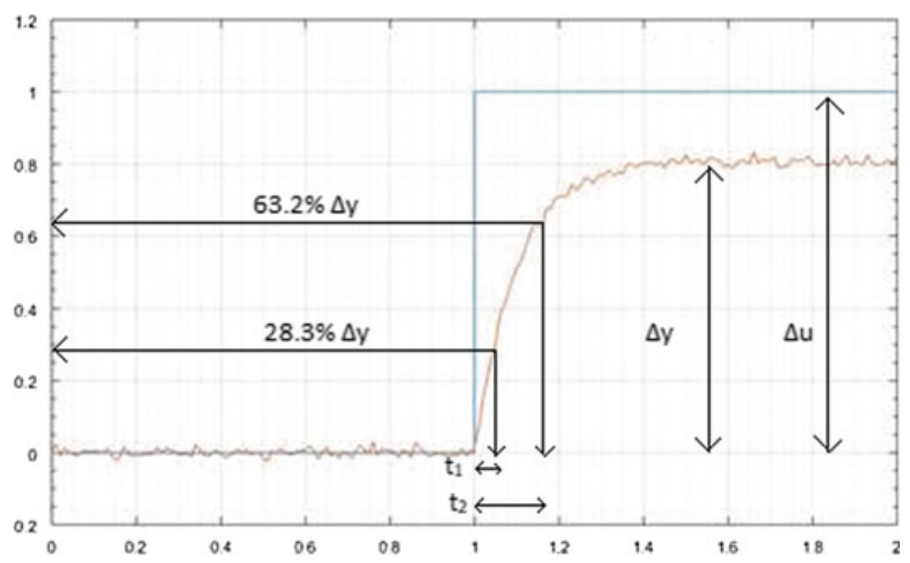

Fig. 2 Sample DC motor behaviour plot

The computed values are fed in the FOPTD model of the motor and from the schematic obtained in the SIMULINK (refer Fig. 3), and a transfer function graph of the DC motor is acquired [5].

In the proposed system, from Eq. (1), the term $d$, i.e. time delay is eliminated since the value derived from the transfer function graph is insignificant (refer Fig. 4). Also, the transfer function acquired from the reaction curve method is in time domain, i.e. continuous transfer function. It is further converted to discrete form so as to get the same sampling time, and the discrete and continuous elements do not mix within the SIMULINK model to be used in the digital circuit. The sampling frequency used is kept same as that in the input.

A comparison between the actual DC motor response and the transfer function model developed using the above-derived terms is plotted. It can be observed that the transfer function model developed is a decent fit.
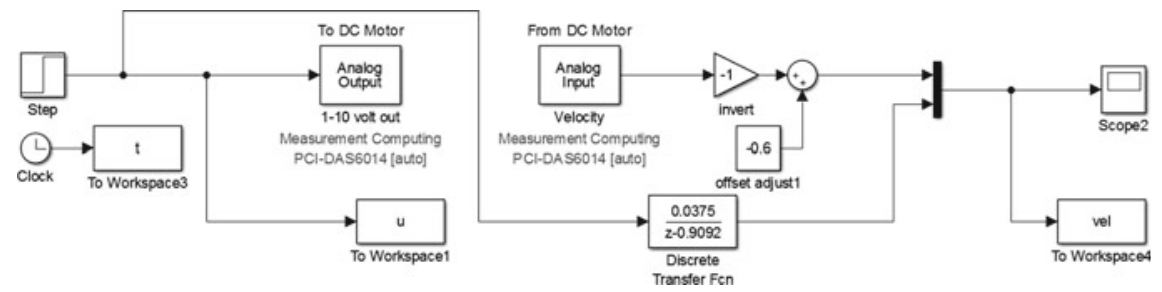

Fig. 3 SIMULINK schematic to acquire TF 


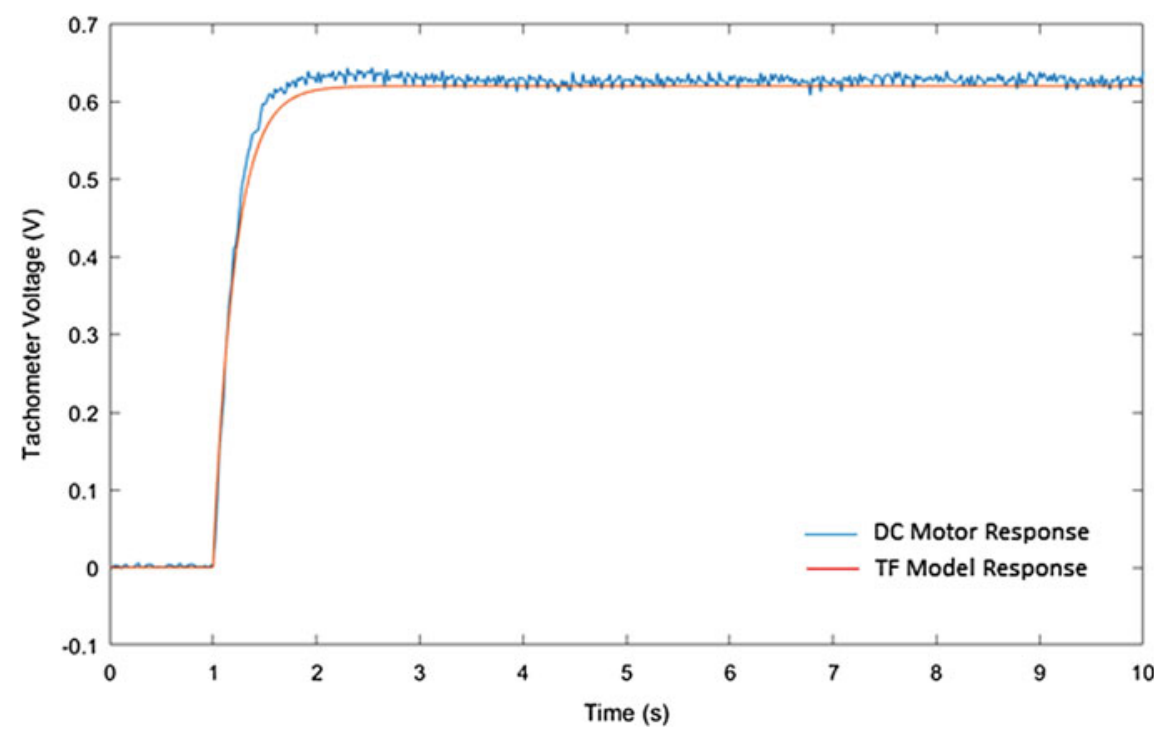

Fig. 4 Transfer function response

\section{Control System Design}

A proportional controller makes the control signal directly proportional to the magnitude of the error signal. One of the main drawbacks of proportional—only control is its inability to leave zero steady-state error. Increasing the gain reduces the offset but can lead to noise problems and instability. Alternatively, by introducing the Integral action in the controller, which perhaps act as an automatic bias adjuster can eliminate the offset without introducing much of instability. But, the downside is that the integral action can actually introduce oscillatory behaviour, and this is eliminated only if the parameter is set correctly. The introduction of an integrator now exhibits dynamic behaviour of its own. Now, the system will have two adjustable parameters $K_{P}$ and $K_{I}$, the proportional and the integral gain.

Using Pade approximation [6, 7], slight variations in the approach of direct design synthesis (DDS), which has achieved widespread industrial acceptance, are used to compute the proportional and integral parameters of the system.

For a FOPTD model with a PI controller, the parameters are derived as follows,

$$
\begin{aligned}
& K_{P}=\frac{2 \tau+d}{2 K \lambda} \\
& K_{I}=\frac{2}{2 \tau+d}
\end{aligned}
$$


Derivative control is highly susceptible to noise. The noise sensitivity is due to high rate of change within the signal when there are noise spikes. Also, in the proposed system, the output of the DC motor consists of fair share of noise. Hence, by incorporating derivative control in the system, entire system can turn unstable. As a matter of fact, the given system attains stability by incorporating PI control only.

\section{Implementation}

\subsection{PI Control Observed Under Disturbances}

The output (speed) of the DC motor is measured and fed back to the PI controller via a closed-loop system. The feedback signal is first compared with the reference signal. An error signal is generated at the summing point by calculating the difference between the actual output and the reference signal by simple subtraction. This error signal is passed through to the PI controller for the required modification set by specification of controller parameters. The control outputs an appropriate control signal such that the error is then eliminated (Fig. 5).

A mechanical disturbance in the form of brake can be applied to the motor. This is analogous/equivalent to an electric bike travelling on a now steeper gradient (i.e. uphill). Due to the incorporation of PI control, it now maintains the speed of the motor at the certain set speed.

\subsection{PI Control Observed Under Varying Pre-defined Speeds}

PI control is also observed whilst setting different set point speeds. The proposed system consists of manual/auto switch. If the switch is on manual mode, the driver will have to control the speed of the motorcycle manually using the throttle. If the switch is on auto mode, a set point speed is decided by the driver, and the motorcycle

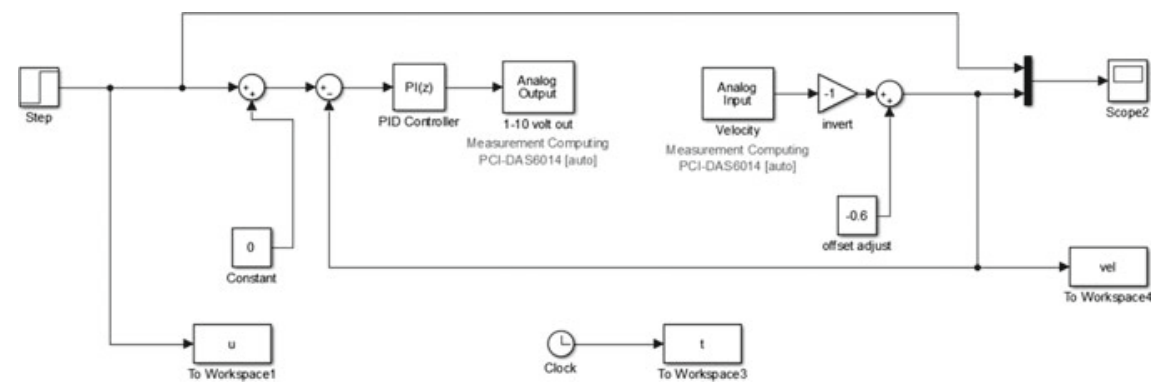

Fig. 5 PI control under disturbances, schematic 


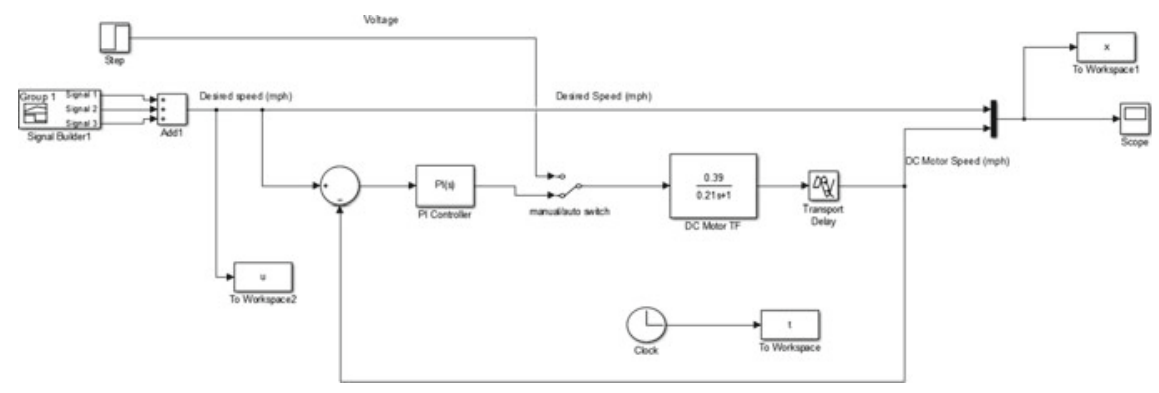

Fig. 6 PI control under varying pre-defined speeds, schematic

will drive at the set speed even under most disturbances. For the purpose of simulation only, the varying set point speed block can be represented by signal builder in the SIMULINK. The varying signals are the step signals of different magnitudes. The PI controller will try to eliminate the error signal as mentioned and match with the new set speeds at earliest (Fig. 6).

\section{Results and Discussions}

\subsection{Disturbance Rejection Capability}

Mechanical disturbance is added to the system in the form of braking. It can be seen that the disturbance is added in the system at $t=5.5 \mathrm{~s}$, and it takes around $4 \mathrm{~s}$ to settle at pre-defined value since $\lambda=4$. The PI controller recovers from this disturbance and adjusts itself to the pre-defined speed. This is the disturbance rejection capability of the PI controller in the system.

\subsection{Set Point Tracking Capability}

Set point tracking capability allows the user to set the set point speeds of the motorcycle. In the proposed system, signal builder allows to set varying amplitude for the step input for the motor (Figs. 7 and 8).

This varying step input does act like different set point speeds for the motorcycle, and PI controller tries to adapt to these speeds. 


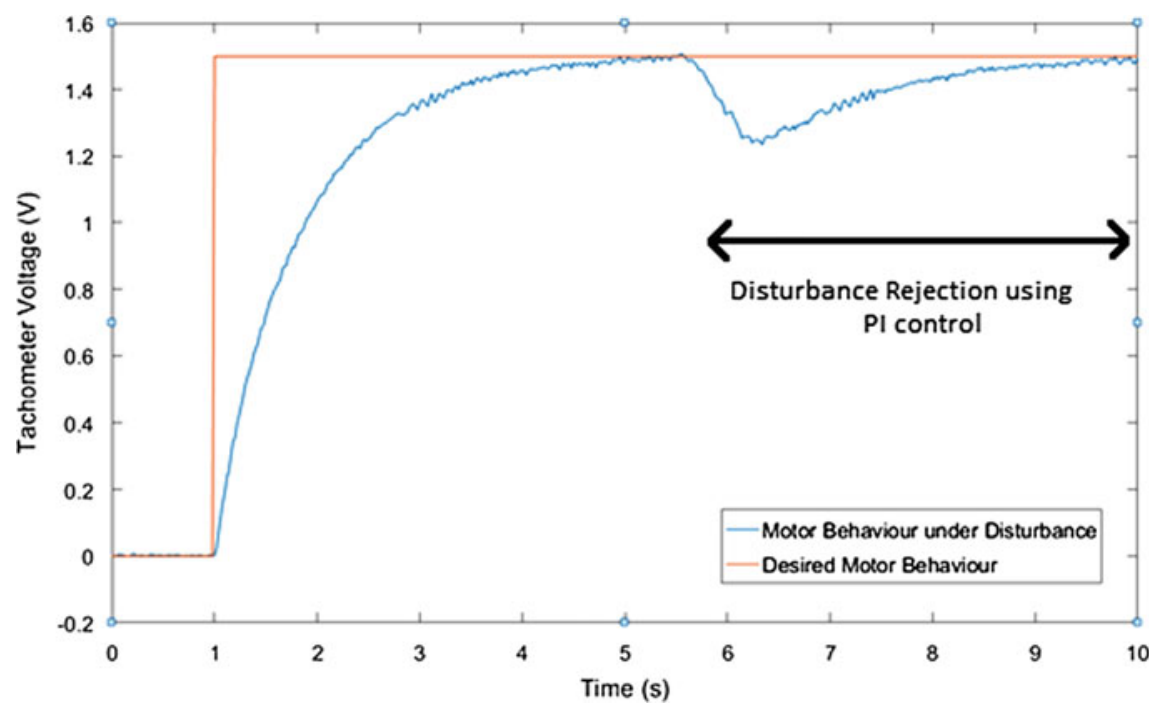

Fig. 7 Disturbance rejection behaviour using PI

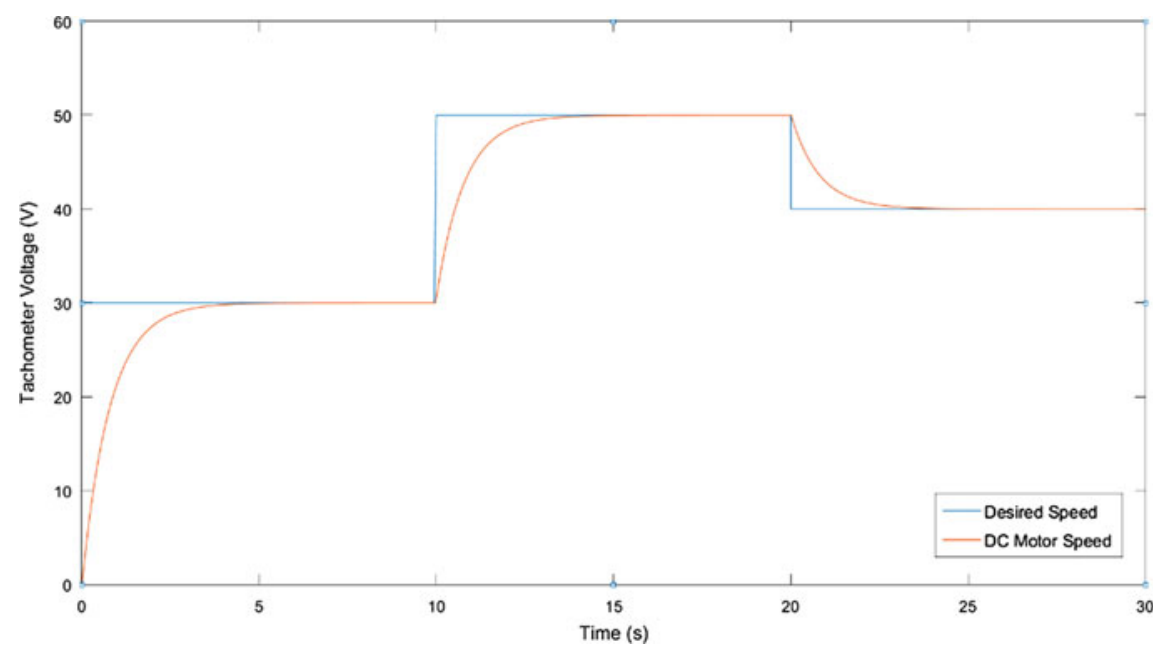

Fig. 8 Set point tracking capability using PI

\section{Conclusions and Future Enhancements}

The transfer function model derived from the reaction curve method finds a decent fit with the motor output. This transfer function can be used as a basis for tuning. PI control approach is incorporated to trace this transfer function model. The proposed 
system can efficiently work in maintaining the speed of the motor and perform the function of cruise control in electric motorcycles.

Induction motors are now used widespread. One such notable example would be four-wheel based electric vehicles. An equivalent mathematical model of an induction motor can be computed using the process defined in the proposed system. Further analysis can be resolved, and a similar cruise control system for four-wheeled system based on induction motors can be devised.

\section{References}

1. Wang JB (2001) Control of electric machinery. Gau Lih Book co., Ltd, Taipei, Taiwan

2. DC motor drives. https://www.engineerlive.com/content/21329

3. Huang G, Lee S (2008) PC-based PID speed control in DC motor. In: International conference on audio, language and image processing, 2008. ICALIP 2008, pp 400-407. IEEE

4. Hanselman DC (2003) Brushless permanent magnet motor design, The Writers' Collective

5. Sung SW, Lee J, Lee IB (2009). Process identification and PID control. Wiley

6. Rivera DE, Morari M, Skogestad S (1986) Internal model control: PID controller design. Ind Eng Chem Process Des Dev 25(1):252-265

7. Al-Mashakbeh ASO (2009) Proportional integral and derivative control of brushless dc motor. Eur J Sci Res 35(2):198-203 